\title{
THE EFFECTS OF PLANT DENSITY AND YEAR ON YIELD OF PURPLE CONEFLOWER (Echinacea purpurea (L.) MOENCH)
}

\author{
Leyla Sezen TANSI ${ }^{*}$, Sengul KARAMAN², Ozlem TONCER ${ }^{3}$, Selin GEDIK ${ }^{1}$ \\ ${ }^{I}$ Çukurova University, Faculty of Agriculture, Department of Field Crops, Balcali-Adana, TURKEY \\ ${ }^{2}$ Sutcu Imam University, Faculty of Science, Department of Biology, Kahramanmaras, TURKEY \\ ${ }^{3}$ Dicle University, Faculty of Agricultural, Department of Field Crops, Diyarbakir, TURKEY.
}

Corresponding author: lsezen@mail.cu.edu.tr

Received: 06.05.2015

\begin{abstract}
Purple coneflower, one of the most popular medicinal plants, is not naturally found in Turkey. Here, Purple coneflower plants were grown in the Cukurova Region of Turkey and the effect of plant density and year on the herb yields were studied. Plants were harvested in June, 2011 and 2012 as second and third seasons of growth in Cukurova. Since flowering plants were not available for one-year-old plants in 2010. Purple coneflower were harvested at the aerial parts when the flowering time at the $2^{\text {nd }}$ and $3{ }^{\text {nd }}$ season, roots were harvested in fall. Although the weight of different aerial parts and roots per plant $\left(\mathrm{g} \mathrm{plant}^{-1}\right)$ were not changed $^{-1}$ according to different plant densities $(30 \times 90,45 \times 90,60 \times 90 \mathrm{~cm})$ these parameters were changed according to the years. Also, fresh weight of herb $\left(g_{\text {plant }}^{-1}\right)$, dry weight of flowers $\left(g\right.$ plant $\left.^{-1}\right)$ and number of main stems (number plant ${ }^{-1}$ ) were not changed in pertinent to years and plant densities. Fresh yield of herb, fresh yield of stem, fresh yield of leaf, dry yields of stem, dry yields of leaf, number of seconder buds $\left(\mathrm{kg} \mathrm{ha}^{-1}\right) \mathrm{had}^{-}$ differences according to plant densities and years.
\end{abstract}

Key words: Echinacea purpurea, plant year, plant density.

\section{INTRODUCTION}

Purple coneflower is widely cultivated for medicinal preparations. Medicinally all parts of the plant may be used, but the leaves and flowers or the root/crown tissues are often extracted and used in capsules and tinctures (Binns, 2002; Li, 1998).

McGregor, (1968) reported the classification system of the genus Echinacea contains nine species and four varieties, all native to North America. Among them, $E$. angustifolia DC., E. purpurea (L.) Moench, and E. pallida have been widely used as dietary supplements (Kindscher et al., 2008; Li, 1998). In recent years, E. purpurea has become the primary species for field cultivation so its product and characteristics were extensively studied. (National Center for Biotechnology Information, 2011). This may be due to less effort is required for its cultivation, resulting from the little or no seed dormancy in commercial seed lots $(\mathrm{Qu}$ et al., 2005; Qu and Wirdlechner, 2012), relatively rapid growth, and broad adaptation to various soil types $(\mathrm{Li}, 1998)$.

The market demand of Purple coneflower material was initially provided by collecting from natural plants. But the increasing uses for herbal products has enhanced the field cultivation of these species the last decade (Dall'Acqua et al., 2010; Li, 1998). Purple coneflower is not naturally found in Turkey but its leaves, flowers and roots from the small scaled cultivation are exported (Cebi, 2013). Although the cultivation of Purple coneflower has been expanded in formation regarding the effects of genetic diversity, growing condition climates and cultivation practices on active constituents (e.g. caffeoyl derivatives) and production of Purple coneflower are still very limited (Chen et al., 2008). Therefore, this study could be important in terms of its agronomic management, to maximize yield in field cultivation.

In addition determination as the effects of plant density and plant maturity at harvest of foliage, flowers, and roots on yields is significant as well as, the effects of foliar and flower harvesting on subsequent root development (Callan, 2005).

Purple coneflower is not a good weed competitor in its first year, during the summer months, weed control is critical during the first year; clean cultivation between rows is desirable, with hand weeding within the row as necessary. The ideal field density of Purple coneflower has been controversial, ranging from 6070 to 22258 plants per ha. Some commercial sellers of Purple coneflower 
plugs have advocated much higher planting rates (Anonym, 2009).

The purpose of this study was determined the effect of plant density and year on the yield of different parts of Purple coneflower.

\section{MATERIALS AND METHODS}

\section{Plant material}

Seedlings of Purple coneflower were obtained from the Atatürk Horticultural Central Research Institute, Turkey in 2010.

\section{Methods}

Plants were grown under field conditions, at the Research Station of the in Field Crop Department, Faculty of Agricultural, Çukurova University in Adana from 2010 to 2012. This location, in southern Turkey, has a typical Mediterranean-type climatic conditions (latitude $36^{\circ} 42^{\prime}$ $\mathrm{N}$ and longitude $26^{\circ} 45^{`} \mathrm{E}$ and at $23 \mathrm{~m}$ asl). Mean daily maximum, minimum temperatures and annual rainfall at this site in 2011 and 2012 were $29.3{ }^{\circ} \mathrm{C}, 8.48{ }^{\circ} \mathrm{C}$, and 628 $\mathrm{mm}, \quad 30.2{ }^{\circ} \mathrm{C}, 9.9{ }^{\circ} \mathrm{C}$ and $1034.3 \mathrm{~mm}$ respectively (Anonym, 2013). The means of maximum temperatures and total rainfall from beginning sprouting to flowering period (April-June) in 2011 and 2012 were 31.9, $36{ }^{\circ} \mathrm{C}$ and $180.9,109.8 \mathrm{~mm}$ respectively.

Seedlings were transplanted at three different densities $(30 \times 90,45 \times 90$ and $60 \times 90 \mathrm{~cm})$ into plots $(3.60 \times 6.30 \mathrm{~m}$ sized) on May 20, 2010. Each plot contained seven rows. Plots were hand-weeded and sprinkler irrigated as needed to maintain vigorous plant growth. Flowering is delayed, and occurred in September 2010, at this time, plants was dwarf and the optimum density was not reached. Therefore, data taken from the plants harvested in 2010 were not analyzed. Plants developed were harvested in
June 2011 and 2012 second and third seasons of growth. Plants were harvested by cutting to about $5 \mathrm{~cm}$ above ground level $2^{\text {nd }}$ and $3^{\text {nd }}$ season during in full flowering stage on June 26, 2011 and 2012. So ten plants were harvested from each plot for collecting data. Also, in order to determine two plants from each plot were harvested on November 14, 2011 and 2012 washed and air dried for three weeks in shade. Root harvesting were performed when plants were dormant, when leaves begin to turn brown. Plant lengths were measured from ground to tip in the longest stem at the harvest time. Harvested plants were separated by hand into stems, leaf, flower buds and flowers.

Field trials were arranged in Randomized Complete Block design with 3 replications. The collected data over two years were statistically analyzed using Anova technique according to the split-plot design where plant densities as main plot and years as sub-plot, the means were compared by using the LSD test (Steel and Torrie, 1980). Year was considered as independent factor because Purple coneflower is perennial plant.

\section{RESULTS AND DISCUSSION}

Since, full flowering plants were not available in first year, the data of obtained from the second and the third years were presented here.

The higher fresh and dry biomass weight per plant (except for flowers) with fresh and dry biomass yields per unite area were found for in plant spacing of $30 \times 90 \mathrm{~cm}$ spacing (3703.3 number of plant $\mathrm{da}^{-1}$ ), in the second year (Table 1, 2, 3, 4, 5, 7). Falk et al. (1999) and Kleitz et al. (2003) reported that in row plant spacings of 30.5, 45.7, $61 \mathrm{~cm}$ had higher plot yields of Purple coneflower at the $30.5 \mathrm{~cm}$ spacing (358780 number of plant ha- $\mathrm{h}^{-1}$ ). Yields of herb, stem and leaf were changed significantly depending on the years and plant densities (Table 4).

Table 1. Plant height and fresh weight per plant of different plant parts of Purple coneflower $\left(\mathrm{g} \mathrm{plant}^{-1}\right)$

\begin{tabular}{|c|c|c|c|c|c|c|c|c|c|c|c|c|c|c|c|c|c|c|}
\hline \multirow{3}{*}{$\begin{array}{c}\text { Plant Densities } \\
(\mathrm{cm})\end{array}$} & \multicolumn{3}{|c|}{$\begin{array}{l}\text { Plant Height } \\
(\mathrm{cm})\end{array}$} & \multicolumn{3}{|c|}{$\begin{array}{c}\text { Fresh Weight of Herb } \\
\left(\mathrm{g} \mathrm{plant}^{-1}\right)\end{array}$} & \multicolumn{3}{|c|}{$\begin{array}{c}\text { Fresh Weight of } \\
\text { Flower }\left(\mathrm{g}_{\text { plant }}{ }^{-1}\right)\end{array}$} & \multicolumn{3}{|c|}{$\begin{array}{l}\text { Fresh Weight of } \\
\left.\text { Bud (g plant- }{ }^{1}\right)\end{array}$} & \multicolumn{3}{|c|}{$\begin{array}{c}\text { Fresh Weight of Stem } \\
\left({\left.\text { ( } \text { plant }^{-1}\right)}^{-1}\right.\end{array}$} & \multicolumn{3}{|c|}{$\begin{array}{c}\text { Fresh Weight of Leaf } \\
\left.\text { (g plant }^{-1}\right)\end{array}$} \\
\hline & \multicolumn{2}{|c|}{ Years } & \multicolumn{4}{|c|}{ Years } & \multicolumn{2}{|c|}{ Years } & \multicolumn{4}{|c|}{ Years } & \multicolumn{2}{|c|}{ Years } & \multicolumn{4}{|c|}{ Years } \\
\hline & 2011 & 2012 & Mean & 2011 & 2012 & Mean & 2011 & 2012 & Mean & 2011 & 2012 & Mean & 2011 & 2012 & Mean & 2011 & 2012 & Mean \\
\hline $30 \times 90$ & 114.7 & 96.0 & 105.4 & 941.1 & 567.0 & 754.0 & 221.0 & 165.7 & 193.3 & 36.0 & 16.3 & 26.2 & 395.7 & 218.3 & 307.0 & 288.3 & 154.1 & 221.2 \\
\hline $45 \times 90$ & 111.2 & 94.9 & 103.1 & 882.9 & 645.7 & 764.2 & 205.0 & 163.0 & 184.0 & 38.6 & 25.7 & 32.1 & 383.0 & 243.7 & 313.3 & 256.3 & 215.0 & 235.7 \\
\hline $60 \times 90$ & 108.7 & 91.4 & 100.1 & 921.6 & 676.3 & 798.9 & 250.7 & 202.0 & 226.3 & 35.4 & 28.3 & 31.9 & 374.5 & 234.0 & 304.3 & 261.0 & 192.7 & 226.8 \\
\hline Mean & $111.5 \mathrm{a}$ & $94.1 \mathrm{~b}$ & 102.8 & $915.1 \mathrm{a}$ & $629.7 \mathrm{~b}$ & 772.4 & 225.6 & 176.9 & 201.2 & $36.6 \mathrm{a}$ & $23.4 \mathrm{~b}$ & 30.1 & $384.4 \mathrm{a}$ & $232.0 \mathrm{~b}$ & 308.2 & $268.5 \mathrm{a}$ & $187.3 \mathrm{~b}$ & 227.9 \\
\hline LSD (\%5) & 9. & & NS & & & NS & & & NS & & & NS & & & NS & & 4.3 & NS \\
\hline
\end{tabular}

Table 2. Dry weights per plants of different plant parts of Purple coneflower (g plant ${ }^{-1}$ )

\begin{tabular}{|c|c|c|c|c|c|c|c|c|c|c|c|c|c|c|c|}
\hline \multirow[t]{3}{*}{$\begin{array}{c}\text { Plant Densities } \\
(\mathbf{c m})\end{array}$} & \multicolumn{3}{|c|}{$\begin{array}{c}\text { Dry Weight of Herbs } \\
\left(\text { g plant }^{-1}\right)\end{array}$} & \multicolumn{3}{|c|}{$\begin{array}{c}\text { Dry Weight of } \\
\text { Flower }\left(\mathrm{g} \mathrm{plant}^{-1}\right)\end{array}$} & \multicolumn{3}{|c|}{$\begin{array}{c}\text { Dry Weight of Bud } \\
\left(\text { g plant }^{-1}\right)\end{array}$} & \multicolumn{3}{|c|}{$\begin{array}{c}\text { Dry Weight of Stem } \\
\left(\text { g plant }^{-1}\right)\end{array}$} & \multicolumn{3}{|c|}{$\begin{array}{c}\text { Dry Weight of Leaf } \\
\left(\mathrm{g} \text { plant } \mathrm{t}^{-1}\right)\end{array}$} \\
\hline & \multicolumn{2}{|c|}{ Years } & \multirow[b]{2}{*}{ Mean } & \multicolumn{2}{|c|}{ Years } & & \multicolumn{2}{|c|}{ Years } & \multirow[b]{2}{*}{ Mean } & \multicolumn{2}{|c|}{ Years } & \multicolumn{4}{|c|}{ Years } \\
\hline & 2011 & 2012 & & 2011 & 2012 & Mean & 2011 & 2012 & & 2011 & 2012 & Mean & 2011 & 2012 & Mean \\
\hline $30 \times 90$ & 288.8 & 202.0 & 245.4 & 42.7 & 54.0 & 48.4 & 16.0 & 12.7 & 14.3 & 161.4 & 93.7 & 127.6 & 68.7 & 41.7 & 55.2 \\
\hline $45 \times 90$ & 279.1 & 201.0 & 240.1 & 40.7 & 44.3 & 42.5 & 20.9 & 11.7 & 16.3 & 150.8 & 96.7 & 123.7 & 66.8 & 48.7 & 57.7 \\
\hline $60 \times 90$ & 273.6 & 210.0 & 241.8 & 45.5 & 53.3 & 49.4 & 15.8 & 12.3 & 14.1 & 146.5 & 99.0 & 122.8 & 65.8 & 46.0 & 55.9 \\
\hline Mean & $280.5 \mathrm{a}$ & $204.3 \mathrm{~b}$ & 242.4 & 43.0 & 50.6 & 46.8 & $17.5 \mathrm{a}$ & $12.2 \mathrm{~b}$ & 14.9 & $152.9 \mathrm{a}$ & $96.4 \mathrm{~b}$ & 124.7 & $67.0 \mathrm{a}$ & $45.4 \mathrm{~b}$ & 56.3 \\
\hline $\operatorname{LSD}(\% 5)$ & & & NS & & IS & NS & & & NS & & & NS & & & NS \\
\hline
\end{tabular}


Table 3. Numbers of flowers bud, stem and seconder stem of Purple coneflower (number plant ${ }^{-1}$ )

\begin{tabular}{|c|c|c|c|c|c|c|c|c|c|c|c|c|}
\hline Plant Densities & \multicolumn{3}{|c|}{$\begin{array}{l}\text { Numbers of Flowers } \\
\left.\text { (number plant }{ }^{-1}\right)\end{array}$} & \multicolumn{3}{|c|}{$\begin{array}{l}\text { Number of Buds } \\
\left(\text { number plant }{ }^{-1}\right)\end{array}$} & \multicolumn{3}{|c|}{$\begin{array}{c}\text { Number of Main Stems } \\
\left(\text { number plant } t^{-1}\right)\end{array}$} & \multicolumn{3}{|c|}{$\begin{array}{c}\text { Number of Seconder Stems } \\
\left.\text { (number } \text { plant }^{-1}\right)\end{array}$} \\
\hline \multirow{2}{*}{$(\mathrm{cm})$} & \multicolumn{3}{|c|}{ Years } & \multicolumn{3}{|c|}{ Years } & \multicolumn{3}{|c|}{ Years } & \multicolumn{3}{|c|}{ Years } \\
\hline & 2011 & 2012 & Mean & 2011 & 2012 & Mean & 2011 & 2012 & Mean & 2011 & 2012 & Mean \\
\hline $30 \times 90$ & 29.1 & 25.7 & 27.4 & 25.3 & 14.1 & 19.7 & 16.5 & 17.6 & 17.1 & 31.3 & 17.3 & 24.3 \\
\hline $45 \times 90$ & 28.8 & 25.6 & 27.2 & 31.5 & 23.3 & 27.4 & 19.2 & 18.3 & 18.8 & 35.3 & 24.4 & 29.8 \\
\hline $60 \times 90$ & 32.2 & 28.6 & 30.4 & 25.2 & 23.5 & 24.3 & 18.0 & 19.6 & 18.8 & 33.3 & 22.6 & 27.9 \\
\hline Mean & $30.0 \mathrm{a}$ & $26.6 \mathrm{~b}$ & 28.3 & $27.3 \mathrm{a}$ & $20.3 \mathrm{~b}$ & 23.8 & 17.9 & 18.5 & 18.2 & $33.2 \mathrm{a}$ & $21.4 \mathrm{~b}$ & 27.4 \\
\hline $\operatorname{LSD}(\% 5)$ & \multicolumn{3}{|c|}{11.9} & \multicolumn{3}{|c|}{4.3} & \multicolumn{2}{|c|}{ NS } & NS & \multicolumn{2}{|c|}{10.1} & NS \\
\hline
\end{tabular}

Table 4. Fresh yield of different plant parts of Purple coneflower $\left(\mathrm{kg} \mathrm{ha}^{-1}\right)$

\begin{tabular}{|c|c|c|c|c|c|c|c|c|c|c|c|c|c|c|c|}
\hline \multirow{3}{*}{$\frac{\text { Plant Densities }}{(\mathrm{cm})}$} & \multicolumn{3}{|c|}{$\begin{array}{c}\text { Fresh Yields of Herb } \\
\left(\mathrm{kg} \mathrm{ha}^{-1}\right)\end{array}$} & \multicolumn{3}{|c|}{$\begin{array}{c}\text { Fresh Yields of Flower } \\
\left(\mathrm{kg} \mathrm{ha}^{-1}\right)\end{array}$} & \multicolumn{3}{|c|}{$\begin{array}{c}\begin{array}{c}\text { Fresh Yields of Bud } \\
\left(\mathrm{kg} \mathrm{ha}_{-}{ }^{1}\right)\end{array} \\
\end{array}$} & \multicolumn{3}{|c|}{$\begin{array}{c}\text { Fresh Yields of Stem } \\
\left(\mathrm{kg} \mathrm{ha}^{-1}\right)\end{array}$} & \multicolumn{3}{|c|}{$\begin{array}{c}\begin{array}{c}\text { Fresh Yields of Leaf } \\
\left(\mathrm{ha}^{-1}\right)\end{array} \\
(\mathrm{kg}\end{array}$} \\
\hline & \multicolumn{3}{|c|}{ Years } & \multicolumn{3}{|c|}{ Years } & \multicolumn{3}{|c|}{ Years } & \multicolumn{3}{|c|}{ Years } & \multicolumn{3}{|c|}{ Years } \\
\hline & 2011 & 2012 & Mean & 2011 & 2012 & Mean & 2011 & 2012 & Mean & 2011 & 2012 & Mean & 2011 & 2012 & Mean \\
\hline $30 \times 90$ & 34854 & 20536 & $27695 a$ & 8185 & 6136 & $7160 \mathrm{a}$ & $1334 \mathrm{a}$ & $605 \mathrm{c}$ & 970 & 14656 & 8086 & $11370 \mathrm{a}$ & 10679 & 5709 & $8193 \mathrm{a}$ \\
\hline $45 \times 90$ & 21800 & 15983 & 18892 b & 5062 & 4025 & $4543 \mathrm{~b}$ & $952 \mathrm{~b}$ & $634 \mathrm{c}$ & 793 & 9457 & 6016 & $7736 \mathrm{ab}$ & 6329 & 5309 & $5818 \mathrm{~b}$ \\
\hline $60 \times 90$ & 17067 & 12167 & $14617 \mathrm{c}$ & 4642 & 3741 & $4191 \mathrm{~b}$ & $656 \mathrm{c}$ & $525 \mathrm{c}$ & 590 & 6935 & 4333 & $5634 b$ & 4833 & 3568 & $4200 \mathrm{~b}$ \\
\hline Mean & $24574 \mathrm{a}$ & $16229 \mathrm{~b}$ & 20401 & 5963 & 4634 & 5298 & $981 \mathrm{a}$ & $588 \mathrm{~b}$ & 784 & 10349 a & $6145 \mathrm{ab}$ & 8247 & $7281 \mathrm{a}$ & $4862 \mathrm{~b}$ & 6071 \\
\hline $\operatorname{LSD}(\% 5)$ & \multicolumn{2}{|c|}{1049.0} & 416.0 & \multicolumn{2}{|c|}{ NS } & 154.1 & \multicolumn{2}{|c|}{$\begin{array}{c}12.4 \\
\text { (int) } 21.44\end{array}$} & NS & \multicolumn{2}{|c|}{686.0} & 447.4 & \multicolumn{2}{|c|}{187.0} & 179.3 \\
\hline
\end{tabular}

In calculation from Table 1 and Table 7, The higher weight of fresh herb + root $\left(4.1 \mathrm{~kg} \mathrm{~m}^{-2}\right)$ was found for spacing $30 \times 90 \mathrm{~cm}\left(3.7\right.$ plant $\left.\mathrm{m}^{-2}\right)$, in the second year. Galambosi (1992) reported fresh biomass (herb + root) as $4.5 \mathrm{~kg} \mathrm{~m}^{-2}$ for spacing $40 \times 40 \mathrm{~cm}\left(6-7\right.$ plant $\left.\mathrm{m}^{2}\right)$. Fresh biomass decreased significantly with increased in intra row-spacing from 30 to $60 \mathrm{~cm}$ (Table 4). Callan et al. (2005) have stated that very dense plant populations (over 15 plants per $\mathrm{m}^{2}$ ) resulted in high biomass production.
Plant height $(\mathrm{cm})$ and fresh herb weight $\left(\mathrm{g}\right.$ plant $\left.{ }^{-1}\right)$ were decreased significantly in 2012 as compared to second year (2011) (Table1). Galambosi et al. (1992) reported that Purple coneflower was cultivated as a biennial plant in Finland. Plant height in third years could be adversely affected by maximum temperature of, $53{ }^{\circ} \mathrm{C}$ in June 2012 (Anonym, 2013). The higher plant height $(115 \mathrm{~cm})$ was obtained due to abundant rainfall in AprilJune months in 2011 .

Table 5. Dry yield of different plant parts of Purple coneflower $\left(\mathrm{kg} \mathrm{ha}^{-1}\right)$

\begin{tabular}{|c|c|c|c|c|c|c|c|c|c|c|c|c|c|c|c|}
\hline \multirow{3}{*}{$\begin{array}{c}\text { Plant Densities } \\
(\mathrm{cm})\end{array}$} & \multicolumn{3}{|c|}{$\begin{array}{c}\text { Dry Yields of Herb } \\
\left(\mathrm{kg} \mathrm{ha}^{-1}\right)\end{array}$} & \multicolumn{3}{|c|}{$\begin{array}{c}\text { Dry Yields of Flower } \\
\left(\mathrm{kg} \mathrm{ha}^{-1}\right)\end{array}$} & \multicolumn{3}{|c|}{$\begin{array}{l}\text { Dry Yields of Bud } \\
\left(\mathrm{kg} \mathrm{ha}^{-1}\right)\end{array}$} & \multicolumn{3}{|c|}{$\begin{array}{c}\text { Dry Yields of Stem } \\
\left(\mathrm{kg} \mathrm{ha}^{-1}\right)\end{array}$} & \multicolumn{3}{|c|}{$\begin{array}{c}\text { Dry Yields of Leaf } \\
\left(\mathrm{kg} \mathrm{ha}^{-1}\right)\end{array}$} \\
\hline & \multicolumn{2}{|c|}{ Years } & \multirow[b]{2}{*}{ Mean } & \multicolumn{2}{|c|}{ Years } & & \multicolumn{2}{|c|}{ Years } & \multirow[b]{2}{*}{ Mean } & \multicolumn{2}{|c|}{ Years } & & \multicolumn{2}{|c|}{ Years } & \multirow[b]{2}{*}{ Mean } \\
\hline & 2011 & 2012 & & 2011 & 2012 & Mean & 2011 & 2012 & & 2011 & 2012 & Mean & 2011 & 2012 & \\
\hline $30 \times 90$ & 10696 & 7481 & $9088 \mathrm{a}$ & 1581 & 2000 & $1791 \mathrm{a}$ & 591 & 469 & $530 \mathrm{a}$ & 5979 & 3469 & $4724 a$ & 2544 & 1543 & $2043 a$ \\
\hline $45 \times 90$ & 6892 & 4963 & $5927 \mathrm{~b}$ & 1005 & 1095 & $1050 \mathrm{~b}$ & 515 & 288 & $401 \mathrm{a}$ & 3723 & 2387 & $3055 \mathrm{~b}$ & 1649 & 1202 & $1425 \mathrm{~b}$ \\
\hline $60 \times 90$ & 5067 & 3889 & $4477 \mathrm{c}$ & 842 & 988 & $914 \mathrm{~b}$ & 293 & 228 & $260 \mathrm{~b}$ & 2714 & 1833 & $2273 \mathrm{~b}$ & 1219 & 852 & $1035 \mathrm{c}$ \\
\hline Mean & 7552 & 5444 & 6487 & 1143 & 1361 & 1252 & 466 & 329 & 397 & 4139 a & $2563 \mathrm{~b}$ & 3351 & $1804 \mathrm{a}$ & $1199 \mathrm{~b}$ & 1501 \\
\hline LSD (\%5) & $\mathrm{N}$ & & 131.9 & & & 31.7 & & & 13.6 & & & 85.8 & & & 38.8 \\
\hline
\end{tabular}

Table 6. Numbers of flowers, bud, stem and seconder stem of Purple coneflower (number ha-1)

\begin{tabular}{|c|c|c|c|c|c|c|c|c|c|c|c|c|}
\hline \multirow{3}{*}{$\begin{array}{c}\text { Plant Densities } \\
(\mathbf{c m})\end{array}$} & \multicolumn{3}{|c|}{$\begin{array}{c}\text { Numbers of Flowers } \\
(\text { number ha-1) }\end{array}$} & \multicolumn{3}{|c|}{$\begin{array}{l}\text { Number of Buds } \\
\left.\text { (number ha } \text { ha }^{-1}\right)\end{array}$} & \multicolumn{3}{|c|}{$\begin{array}{c}\text { Number of Main Stems } \\
\left(\text { number } \mathrm{ha}^{-1}\right)\end{array}$} & \multicolumn{3}{|c|}{$\begin{array}{c}\text { Number of Seconder Buds } \\
\left(\text { number ha' } h^{-1}\right)\end{array}$} \\
\hline & \multicolumn{2}{|c|}{ Years } & \multicolumn{4}{|c|}{ Years } & \multicolumn{2}{|c|}{ Years } & \multicolumn{4}{|c|}{ Years } \\
\hline & 2011 & 2012 & Mean & 2011 & 2012 & Mean & 2011 & 2012 & Mean & 2011 & 2012 & Mean \\
\hline $30 \times 90$ & 1076543 & 953086 & $1014802 \mathrm{a}$ & $937037 \mathrm{a}$ & $522222 \mathrm{c}$ & 729567 & 612346 & 650617 & $631433 \mathrm{a}$ & 1157967 & 641933 & $899950 \mathrm{a}$ \\
\hline $45 \times 90$ & 711934 & 632922 & $672400 \mathrm{~b}$ & $776954 \mathrm{~b}$ & $576132 \mathrm{c}$ & 676500 & 473251 & 452675 & $462917 \mathrm{ab}$ & 870533 & 602400 & $736467 \mathrm{~b}$ \\
\hline $60 \times 90$ & 595679 & 529012 & $562300 \mathrm{~b}$ & $466666 \mathrm{c}$ & $434568 \mathrm{c}$ & 450567 & 332716 & 363580 & $348083 \mathrm{~b}$ & 616049 & 417867 & $516933 \mathrm{c}$ \\
\hline Mean & 794719 & 705007 & 749834 & $726886 \mathrm{a}$ & $510974 \mathrm{~b}$ & 618878 & 472771 & 4888957 & 480811 & $881500 \mathrm{a}$ & $554067 \mathrm{~b}$ & 717783 \\
\hline LSD (\%5) & \multicolumn{2}{|c|}{ NS } & 27910.0 & \multicolumn{2}{|c|}{$\begin{array}{c}9187.0 \\
\text { (int) } 15910.0\end{array}$} & NS & \multicolumn{2}{|c|}{ NS } & 18900.0 & \multicolumn{2}{|c|}{30200.0} & 16230.0 \\
\hline
\end{tabular}

The numbers of main and secondary stems per unite area changed significantly as influenced by plant density although the numbers of main and secondary stems per plant were not changed (Table 3,6 ). The average of main stem two years (18.2 number plant ${ }^{-1}$ ) was similar to the value reported by Starman et al. (1995). E. purpurea grows taller (to $150 \mathrm{~cm}$ ), branches more and has wider leaves than E. angustifolia and E. pallida (Hobbs, 1989).
The minimum acceptable stem length for marketing as a cut flower was reported by Barr (1992) as $40.6 \mathrm{~cm}$.

We observed a few plants infected with Aster yellows disease in our experiment in second and third years. Aster yellows disease is more likely to be recognized in second or third year crops. Symptoms include yellowing or reddish-tinged foliage, stunting, and abnormal flowering, with flowers becoming malformed, and losing their purple pigment (Anonym, 2009; Muller et al., 1973). Aster 
yellow did not damage at harvesting time in June in the Çukurova conditions but aster yellow type mycoplasmalike organisms caused damage only aerial part of plants towards to the last mid-August with high temperature and moisture.

Table 7. Yields and weight of roots of Purple coneflower $\left(\mathrm{g} \mathrm{plant}^{-1}\right)$

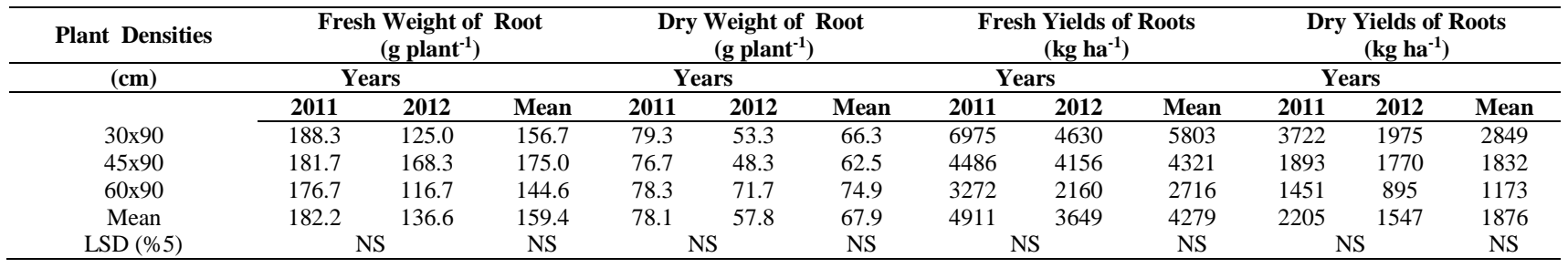

The higher the number of flowers (101480.2 number $\left.\mathrm{ha}^{-1}\right)$, yields of fresh $\left(7160 \mathrm{~kg} \mathrm{ha}^{-1}\right)$ and dry flowers (1791 $\mathrm{kg} \mathrm{ha}^{-1}$ ) per unite area, weight of fresh and dry flowers per plant were found for the $30 \mathrm{~cm}$ in spacing (Table 4, 5, 6). Similar tendency for the effect of plant spacing $(30 \mathrm{~cm}$ spacing) at yields of flowers were reported by Kleitz et al. (2003).

The mean of fresh roots in the second year $(4911 \mathrm{~kg}$ $\mathrm{ha}^{-1}$ ) was higher than (3649 $\left.\mathrm{kg} \mathrm{ha}^{-1}\right)$ value of third years while yields and weight of fresh roots of Purple coneflower were not changed significantly for years and spacing (Table 7). Since they grow underground and smaller roots may break off and remain in the soil, decreasing biomass, as reported (Kleitz et al., 2003). Plant density can have a major effect on plant form (Harper, 1977), and these effects can change the root proportions (Parmenter, 1997).

The higher root yield $\left(5803 \mathrm{~kg} \mathrm{ha}^{-1}\right)$ was found for spacing $30 \times 90 \mathrm{~cm}$ (Table 7). This indicated that root yield could be increased by increasing plant density above the 8 plants per $\mathrm{m}^{2}$ as recommended in Germany (Anonymous, 1986). However, although high plant density maximizes yield, and is likely to help suppress weeds, it carries some risks (Parmenter, 1997). High plant densities increased the danger of fungal rots such as those caused by Sclerotinia spp., especially in combination with heavy soils, poor drainage in the absence of beds or of ridging, or wet and humid conditions (Fry, 1982). But in our conditions number of plant per plot did not reached the risky levels.

\section{CONCLUSION}

Purple coneflower can be grown successfully under the Cukurova conditions. Based on the results of this study under the Cukurova conditions, $(30 \times 90 \mathrm{~cm})$ spacing of Purple coneflower resulted in the higher yields in comparing to the $45 \times 90 \mathrm{~cm}$ and the $60 \times 90 \mathrm{~cm}$ spacings. Therefore, high plant density or narrow plant spacing could be recommended for high plant yield. Under the Cukurova conditions where the Mediterranean type climate prevail with has hot and drought summer and mild rainy winters consequently, growth rates were accelerated and covered spacing of $30 \times 90 \mathrm{~cm}$ but, at the other plant spacing the same effect was not observed such as, plants did not covered inter rows spacing even in the later growing years.

\section{LITERATURE CITED}

Anonymous. 1986. Cultivation of Echinacea. Heil und Gewürzpflanzen 3.1.

Anonym. 2009. Provincial Specialist, Specialty Crops, Crops Branch Saskatchewan Ministry of Agriculture, October.

Anonym. 2013. Climatical Data of Adana Province State Meteorological Station Statistics, Adana.

Barr C. 1992. The kindest cuts of all: How to evaluate new crops. Greenhouse Manager. 11:82-84.

Binns S. E., J. F. Livesey, J.T. Arnason, and B. R. Baum. 2002. Phytochemical variation in Echinacea from roots and flower heads of wild and cultivated populations. J Agric Food Chem. 50: 3673-87.

Callan N. W., T. Yokelson, S. Wall-MacLane, M. P. Westcott, J. B. Miller, and G. Ponder. 2005. Seasonal trends and plant density effects on cichoric acid in Echinacea purpurea (L.) Moench. Journal of Herbs, Spices \& Medicinal Plants, 11: 35-46.

Cebi, H. 2013. Cultivation of Echinacea in Manisa. Manisa İl Tarım ve Hayvancılık Müdürlüğü.

Chen S. C. Zhang and J. M. Sung. 2008. Biomass and caffeoyl phenols production of Echinacea purpurea grown in Taiwan. Expl Agric., 44: 497-507.

Dall'Acqua, S., N. Aiello, F. Scartezzini, V. Albertin, G. Innocenti. 2010. Analysis of highly secondary-metabolite producing roots and flowers of two Echinacea angustifolia DC var. angustifolia accessions. Ind. Crops Prod. 3: 466468.

Falk C. L., H. Voorthuizen, M. M. Wall, S. J. Guldan, C. A. Martin, K. M. Kleitz. 1999. Costs and returns of growing selected medicinal herbs in New Mexico indicate positive return to land and risk likely. Hort Technology, 9: 681-686.

Fry W. E. 1982. Principles of plant disease management. New York, Academic Press.

Galambosi B. 1992. Introduction of Echinacea purpurea and Leuza carthamoisdes into cultivation in Finland. Acta Hort. 208:69-72.

Harper J. L. 1977. Population Biology of Plants, London, Academic Press.

Hobbs C. 1989. The Echinacea handbook. Eclectic medical publications, Portland, OR.p.1-7.

Kindscher K., D.M. Price and L. Castle. 2008. Resprouting of Echinacea angustifolia augments sustainability of wild medicinal plant population. Econ Bot. 62:139-147.

Kleitz K. M., M M. Wall, C.L. Falk, C.A. Martin, M. D. Remmenga, S. J.Guldan. 2003. Yield potential of selected medicinal herbs grown at three plant spacings in New Mexico. Hort Technology., 13: 631-636.

Li, T.S.C. 1998. Echinacea, cultivation and medicinal value, Hort Technology 8:122-129. 
Muller H. M., H. Kleinhempel, D. Spaar, and H. J.Muller. 1973. Mycoplasma-like organisms in ornamental plants with flower phyllody symptoms. Archiv. Fur Phytopath. Und Pflanz., 9: 95-104.

McGregor R. L. 1968. The taxonomy of the genus Echinacea (Compositae). The University of Kansas Science Bulletin, 48: 113-142.

National Center for Biotechnology Information, 2011. PubMed database. Accessed http://www.ncbi.nlm.nih.gov/pubmed/on.

Parmenter G. A., R.P., Littlejohn, 1997. Planting density effects on root yield of Purple coneflower (Echinacea purpurea (L.) Moench). New Zealand Journal of Crop and Horticultural Science, Vol. 25:169-175.

Starman T.W., T. A. Cerny and A. J. MacKenzie.1995. Productivity and profitability of some field-grown specialty cut flowers, Hort Science, 30:1217-1220.
Steel, R.G.D. and J.H. Torrie, 1980. Principles and Procedures of Statistics: a Biometrical Approach. Second Edition. New York: McGraw-Hill Kogakusha.

Seidler-Lozykowska K. and J. Dabrowska. 2003. Yield and polyphenolic acids content in purple coneflower (Echinacea purpurea Moench ) at different growth stages, Journal of Herbs, Spices \& Medicinal Plants, 10:3, 7-12.

Qu L., X. Wang, Y. Chen, R. Scalzo, M.P., Widrlechner, J. Davis, and J. Hancock. 2005. Commercial seed lots exhibit reduced seed dormancy in comparison to wild seed lots of Echinacea purpurea. Hort Science, 40:1843-1845.

Qu L. and M.P., Widrlechner, 2012. Reduction of seed dormancy in Echinacea pallida (Nutt.) by in-dark seed selection and breeding, Ind Crops Prod. 36 (1):88-93. 